\title{
Certain Subclasses of Starlike Functions of Complex Order Involving Generalized Hypergeometric Functions
}

\author{
G. Murugusundaramoorthy ${ }^{1}$ and N. Magesh $^{2}$ \\ ${ }^{1}$ School of Advanced Sciences, VIT University, Vellore 632014, India \\ ${ }^{2}$ Department of Mathematics, Government Arts College (Men), Krishnagiri 635001, India
}

Correspondence should be addressed to G. Murugusundaramoorthy, gmsmoorthy@yahoo.com

Received 24 November 2009; Accepted 18 March 2010

Academic Editor: Stanisława R. Kanas

Copyright (C) 2010 G. Murugusundaramoorthy and N. Magesh. This is an open access article distributed under the Creative Commons Attribution License, which permits unrestricted use, distribution, and reproduction in any medium, provided the original work is properly cited.

Making use of the generalized hypergeometric functions, we define a new subclass of uniformly convex functions and a corresponding subclass of starlike functions with negative coefficients and obtain coefficient estimates, extreme points, the radii of close-to-convexity, starlikeness and convexity, and neighborhood results for the class $T S_{m}^{l}(\alpha, \beta, \gamma)$. In particular, we obtain integral means inequalities for the function $f$ that belongs to the class $T S_{m}^{l}(\alpha, \beta, \gamma)$ in the unit disc.

\section{Introduction}

Let $\mathcal{A}$ denote the class of functions of the form

$$
f(z)=z+\sum_{n=2}^{\infty} a_{n} z^{n}
$$

which are analytic in the open unit $\operatorname{disc} U=\{z: z \in \mathbb{C},|z|<1\}$ and normalized by $f(0)=$ $f^{\prime}(0)-1=0$. Also denote by $T$ the subclass of $\mathbb{A}$ consisting of functions of the form

$$
f(z)=z-\sum_{n=2}^{\infty}\left|a_{n}\right| z^{n}
$$

introduced and studied by Silverman [1]. For functions $f \in \mathcal{A}$ given by (1.1) and $g \in \mathcal{A}$ given by $g(z)=z+\sum_{n=2}^{\infty} b_{n} z^{n}$, it is known [2] the definition of the Hadamard product 
(or Convolution) of $f$ and $g$ by

$$
(f * g)(z)=z+\sum_{n=2}^{\infty} a_{n} b_{n} z^{n}, \quad z \in U
$$

For positive real values of $\alpha_{1}, \ldots, \alpha_{l}$ and $\beta_{1}, \ldots, \beta_{m}\left(\beta_{j} \neq 0,-1, \ldots ; j=1,2, \ldots, m\right)$ the generalized hypergeometric function ${ }_{l} F_{m}(z)$ is defined by

$$
\begin{array}{r}
{ }_{l} F_{m}(z) \equiv{ }_{l} F_{m}\left(\alpha_{1}, \ldots, \alpha_{l} ; \beta_{1}, \ldots, \beta_{m} ; z\right):=\sum_{n=0}^{\infty} \frac{\left(\alpha_{1}\right)_{n} \cdots\left(\alpha_{l}\right)_{n}}{\left(\beta_{1}\right)_{n} \cdots\left(\beta_{m}\right)_{n}} \frac{z^{n}}{n !} \\
\left(l \leq m+1 ; l, m \in N_{0}:=N \cup\{0\} ; z \in U\right),
\end{array}
$$

where $N$ denotes the set of all positive integers and $(\lambda)_{k}$ is the Pochhammer symbol defined by

$$
(\lambda)_{n}= \begin{cases}1, & n=0, \\ \lambda(\lambda+1)(\lambda+2) \cdots(\lambda+n-1), & n \in N .\end{cases}
$$

The notation ${ }_{l} F_{m}$ is quite useful for representing many well-known functions such as the exponential, the Binomial, the Bessel, the Laguerre polynomial, and others; for example, see [3].

Let $H\left(\alpha_{1}, \ldots, \alpha_{l} ; \beta_{1}, \ldots, \beta_{m}\right): \mathcal{A} \rightarrow \mathcal{A}$ be a linear operator defined by

$$
\begin{aligned}
H\left(\alpha_{1}, \ldots, \alpha_{l} ; \beta_{1}, \ldots, \beta_{m}\right) f(z): & z_{l} F_{m}\left(\alpha_{1}, \alpha_{2}, \ldots, \alpha_{l} ; \beta_{1}, \beta_{2}, \ldots, \beta_{m} ; z\right) * f(z) \\
& =z+\sum_{n=2}^{\infty} \Gamma_{n} a_{n} z^{n},
\end{aligned}
$$

where

$$
\Gamma_{n}=\frac{\left(\alpha_{1}\right)_{n-1} \cdots\left(\alpha_{l}\right)_{n-1}}{\left(\beta_{1}\right)_{n-1} \cdots\left(\beta_{m}\right)_{n-1}} \frac{1}{(n-1) !},
$$

unless otherwise stated. For notational simplicity, we can use a shorter notation $H_{m}^{l}\left[\alpha_{1}\right]$ for $H\left(\alpha_{1}, \ldots, \alpha_{l} ; \beta_{1}, \ldots, \beta_{m}\right)$ in the sequel. The linear operator $H_{m}^{l}\left[\alpha_{1}\right]$ is called Dziok-Srivastava operator (see [3]), includ (as its special cases) various other linear operators introduced and studied by Carlson and Shaffer [4], Owa [5], Ruscheweyh [2], and Srivastava and Owa [6]. Motivated by Goodman [7, 8], Rønning [9, 10] introduced and studied the following subclasses of $\mathcal{A}$. A function $f \in \mathcal{A}$ is said to be in the class $S_{p}(\alpha, \beta)$, uniformly $\beta$-starlike functions if it satisfies the condition

$$
\operatorname{Re}\left\{\frac{z f^{\prime}(z)}{f(z)}-\alpha\right\}>\beta\left|\frac{z f^{\prime}(z)}{f(z)}-1\right|, \quad(-1<\alpha \leq 1 ; \beta \geq 0) z \in U
$$


and is said to be in the class $\operatorname{UCV}(\alpha, \beta)$, uniformly $\beta$-convex functions if it satisfies the condition

$$
\operatorname{Re}\left\{1+\frac{z f^{\prime \prime}(z)}{f^{\prime}(z)}-\alpha\right\}>\beta\left|\frac{z f^{\prime \prime}(z)}{f^{\prime}(z)}\right|, \quad(-1<\alpha \leq 1 ; \beta \geq 0) z \in U
$$

Indeed it follows from (1.8) and (1.9) that

$$
f \in \mathrm{UCV}(\alpha, \beta) \Longleftrightarrow z f^{\prime} \in S_{p}(\alpha, \beta)
$$

The interesting geometric properties of these function classes were extensively studied by Kanas et al., in [11-14]. Motivated by Altintas et al. [15], Murugusundaramoorthy and Srivastava [16], and Murugusundaramoorthy and Magesh [17], now, we define a new subclass uniformly starlike functions of complex order.

For $-1 \leq \alpha<1, \quad \beta \geq 0$, and $\gamma \in \mathbb{C} \backslash\{0\}$, we let $T S_{m}^{l}(\alpha, \beta, \gamma)$ be the class of functions $f$ satisfying (1.2) with the analytic criterion

$$
\operatorname{Re}\left\{1+\frac{1}{\gamma}\left(\frac{z\left(H_{m}^{l}\left[\alpha_{1}\right] f(z)\right)^{\prime}}{H_{m}^{l}\left[\alpha_{1}\right] f(z)}-\alpha\right)\right\}>\beta\left|1+\frac{1}{\gamma}\left(\frac{z\left(H_{m}^{l}\left[\alpha_{1}\right] f(z)\right)^{\prime}}{H_{m}^{l}\left[\alpha_{1}\right] f(z)}-1\right)\right|, \quad z \in U
$$

where $H_{m}^{l}\left[\alpha_{1}\right] f(z)$ is given by (1.6).

By suitably specializing the values of $l, m, \alpha_{1}, \alpha_{2}, \ldots, \alpha_{l}, \beta_{1}, \beta_{2}, \ldots, \beta_{m}, \alpha, \gamma$, and $\beta$ the class $T S_{m}^{l}(\alpha, \beta, \gamma)$, leads to various new subclasses of starlike functions of complex order. As for illustrations, we present some examples for the cases.

Example 1.1. If $l=2, m=1$ with $\alpha_{1}=1, \alpha_{2}=1, \beta_{1}=1$, and $f$ of the form of (1.2), then

$$
T \mathbb{S}(\alpha, \beta, \gamma):=\left\{\operatorname{Re}\left\{1+\frac{1}{\gamma}\left(\frac{z f^{\prime}(z)}{f(z)}-\alpha\right)\right\}>\beta\left|1+\frac{1}{\gamma}\left(\frac{z f^{\prime}(z)}{f(z)}-1\right)\right|, z \in U\right\}
$$

Example 1.2. If $l=2, m=1$ with $\alpha_{1}=\delta+1(\delta>-1), \alpha_{2}=1, \beta_{1}=1$, and $f$ of the form of (1.2), then

$$
T R_{\delta}(\alpha, \beta, \gamma):=\left\{\operatorname{Re}\left\{1+\frac{1}{\gamma}\left(\frac{z\left(D^{\delta} f(z)\right)^{\prime}}{D^{\delta} f(z)}-\alpha\right)\right\}>\beta\left|1+\frac{1}{\gamma}\left(\frac{z\left(D^{\delta} f(z)\right)^{\prime}}{D^{\delta} f(z)}-1\right)\right|, z \in U\right\},
$$

where $D^{\delta}$ is called Ruscheweyh derivative of order $\delta(\delta>-1)$ defined by

$$
D^{\delta} f(z):=\frac{z}{(1-z)^{\delta+1}} * f(z) \equiv H_{1}^{2}(\delta+1,1 ; 1) f(z)
$$


Example 1.3. If $l=2, m=1$ with $\alpha_{1}=\mu+1(\mu>-1), \alpha_{2}=1, \beta_{1}=\mu+2$, and $f$ of the form of (1.2), then

$$
T B_{\mu}(\alpha, \beta, \gamma)=\left\{\operatorname{Re}\left\{1+\frac{1}{\gamma}\left(\frac{z\left(J_{\mu} f(z)\right)^{\prime}}{J_{\mu} f(z)}-\alpha\right)\right\}>\beta\left|1+\frac{1}{\gamma}\left(\frac{z\left(J_{\mu} f(z)\right)^{\prime}}{J_{\mu} f(z)}-1\right)\right|, z \in U\right\},
$$

where $J_{\mu}$ is a Bernardi operator [18] defined by

$$
J_{\mu} f(z):=\frac{\mu+1}{z^{\mu}} \int_{0}^{z} t^{\mu-1} f(t) d t \equiv H_{1}^{2}(\mu+1,1 ; \mu+2) f(z)
$$

Example 1.4. If $l=2, m=1$ with $\alpha_{1}=a(a>0), \alpha_{2}=1, \beta_{1}=c(c>0)$, and $f$ of the form of (1.2), then

$$
\begin{aligned}
T L_{c}^{a}(\alpha, \beta, \gamma) & :=\left\{\operatorname{Re}\left\{1+\frac{1}{\gamma}\left(\frac{z(L(a, c) f(z))^{\prime}}{L(a, c) f(z)}-\alpha\right)\right\}\right. \\
& \left.>\beta\left|1+\frac{1}{\gamma}\left(\frac{z(L(a, c) f(z))^{\prime}}{L(a, c) f(z)}-1\right)\right|, z \in U\right\},
\end{aligned}
$$

where $L(a, c)$ is a well-known Carlson-Shaffer linear operator [4] defined by

$$
L(a, c) f(z):=\left(\sum_{k=0}^{\infty} \frac{(a)_{k}}{(c)_{k}} z^{k+1}\right) * f(z) \equiv H_{1}^{2}(a, 1 ; c) f(z) .
$$

Also $T L_{c}^{a}(\alpha, \beta, \gamma)$ was studied by Murugusundaramoorthy and Magesh [19].

The main object of this paper is to study some usual properties of the geometric function theory such as the coefficient bound, extreme points, radii of close to convexity, starlikeness, and convexity for the class $T S_{m}^{l}(\alpha, \beta, \gamma)$. Further, we obtain neighborhood results and integral means inequalities for aforementioned class.

\section{Basic Properties}

First we obtain the necessary and sufficient condition for functions $f$ in the class $T S_{m}^{l}(\alpha, \beta, \gamma)$.

Theorem 2.1. The necessary and sufficient condition for $f$ of the form of (1.2) to be in the class $T S_{m}^{l}(\alpha, \beta, \gamma)$ is

$$
\sum_{n=2}^{\infty}[(n+|\gamma|)(1-\beta)-(\alpha-\beta)] \Gamma_{n}\left|a_{n}\right| \leq(1-\alpha)+|\gamma|(1-\beta)
$$

where $-1 \leq \alpha<1, \beta \geq 0$, and $\gamma \in \mathbb{C} \backslash\{0\}$. 
Proof. Assume that $f \in T S_{m}^{l}(\alpha, \beta, \gamma)$, then

$$
\begin{gathered}
\operatorname{Re}\left\{1+\frac{1}{r}\left(\frac{z\left(H_{m}^{l}\left[\alpha_{1}\right] f(z)\right)^{\prime}}{H_{m}^{l}\left[\alpha_{1}\right] f(z)}-\alpha\right)\right\}>\beta\left|1+\frac{1}{r}\left(\frac{z\left(H_{m}^{l}\left[\alpha_{1}\right] f(z)\right)^{\prime}}{H_{m}^{l}\left[\alpha_{1}\right] f(z)}-1\right)\right|, \\
\operatorname{Re}\left\{1+\frac{1}{r}\left(\frac{z(1-\alpha)-\sum_{n=2}^{\infty}(n-\alpha) \Gamma_{n}\left|a_{n}\right| z^{n}}{z-\sum_{n=2}^{\infty} \Gamma_{n}\left|a_{n}\right| z^{n}}\right)\right\}>\beta\left|1-\frac{1}{\gamma}\left(\frac{\sum_{n=2}^{\infty}(n-1) \Gamma_{n}\left|a_{n}\right| z^{n}}{z-\sum_{n=2}^{\infty} \Gamma_{n}\left|a_{n}\right| z^{n}}\right)\right| .
\end{gathered}
$$

Letting $z \rightarrow 1^{-}$along the real axis, we have

$$
\left\{1+\frac{1}{|\gamma|}\left(\frac{(1-\alpha)-\sum_{n=2}^{\infty}(n-\alpha) \Gamma_{n}\left|a_{n}\right|}{1-\sum_{n=2}^{\infty} \Gamma_{n}\left|a_{n}\right|}\right)\right\}>\beta\left[1-\frac{1}{|\gamma|}\left(\frac{\sum_{n=2}^{\infty}(n-1) \Gamma_{n}\left|a_{n}\right|}{1-\sum_{n=2}^{\infty} \Gamma_{n}\left|a_{n}\right|}\right)\right] .
$$

Hence, by maximum modulus theorem, the simple computational leads the desired inequality

$$
\sum_{n=2}^{\infty}[(n+|\gamma|)(1-\beta)-(\alpha-\beta)] \Gamma_{n}\left|a_{n}\right| \leq(1-\alpha)+|\gamma|(1-\beta)
$$

Conversely, suppose that (2.1) is true for $z \in U$. Then

$$
\operatorname{Re}\left\{1+\frac{1}{\gamma}\left(\frac{z\left(H_{m}^{l}\left[\alpha_{1}\right] f(z)\right)^{\prime}}{H_{m}^{l}\left[\alpha_{1}\right] f(z)}-\alpha\right)\right\}-\beta\left|1+\frac{1}{\gamma}\left(\frac{z\left(H_{m}^{l}\left[\alpha_{1}\right] f(z)\right)^{\prime}}{H_{m}^{l}\left[\alpha_{1}\right] f(z)}-1\right)\right|>0
$$

if

$$
1+\frac{1}{|\gamma|}\left(\frac{(1-\alpha)-\sum_{n=2}^{\infty}(n-\alpha) \Gamma_{n}\left|a_{n}\right||z|^{n-1}}{1-\sum_{n=2}^{\infty} \Gamma_{n}\left|a_{n}\right||z|^{n-1}}\right)-\beta\left[1-\frac{1}{|\gamma|}\left(\frac{\sum_{n=2}^{\infty}(n-1) \Gamma_{n}\left|a_{n} \| z\right|^{n-1}}{1-\sum_{n=2}^{\infty} \Gamma_{n}\left|a_{n}\right||z|^{n-1}}\right)\right]>0 .
$$

That is if

$$
\sum_{n=2}^{\infty}[(n+|\gamma|)(1-\beta)-(\alpha-\beta)] \Gamma_{n}\left|a_{n}\right| \leq(1-\alpha)+|\gamma|(1-\beta),
$$

which completes the proof.

Corollary 2.2. Let the function $f$ defined by (1.2) belong to $\operatorname{TS}_{m}^{l}(\alpha, \beta, \gamma)$. Then

$$
\left|a_{n}\right| \leq \frac{[(1-\alpha)+|\gamma|(1-\beta)]}{[(n+|\gamma|)(1-\beta)-(\alpha-\beta)] \Gamma_{n}}
$$


$n \geq 2,-1 \leq \alpha<1, \beta \geq 0$ and, $\gamma \in \mathbb{C} \backslash\{0\}$, with equality for

$$
f(z)=z-\frac{[(1-\alpha)+|\gamma|(1-\beta)]}{[(n+|\gamma|)(1-\beta)-(\alpha-\beta)] \Gamma_{n}} z^{n}
$$

Next we state the following theorem on extreme points for the class $T S_{m}^{l}(\alpha, \beta, \gamma)$ without proof.

Theorem 2.3 (Extreme Points). Let

$$
f_{1}(z)=z, \quad f_{n}(z)=z-\frac{[(1-\alpha)+|\gamma|(1-\beta)]}{[(n+|\gamma|)(1-\beta)-(\alpha-\beta)] \Gamma_{n}} z^{n} \quad \text { for } n=2,3,4, \ldots
$$

Then $f \in T S_{m}^{l}(\alpha, \beta, \gamma)$ if and only if $f$ can be expressed in the form $f(z)=\sum_{n=1}^{\infty} \lambda_{n} f_{n}(z)$, where $\lambda_{n} \geq 0$ and $\sum_{n=1}^{\infty} \lambda_{n}=1$.

\section{Close-to-Convexity, Starlikeness, and Convexity}

We determine the radii of close-to-convexity, starlikeness, and convexity results for functions in the class $T S_{m}^{l}(\alpha, \beta, \gamma)$ in the following theorems.

Theorem 3.1. Let $f \in T S_{m}^{l}(\alpha, \beta, \gamma)$. Then $f$ is close-to-convex of order $\delta(0 \leq \delta<1)$ in the disc $|z|<r_{1}$, where

$$
r_{1}=\inf _{n \geq 2}\left[\frac{(1-\delta)}{n} \frac{[(n+|\gamma|)(1-\beta)-(\alpha-\beta)]}{[(1-\alpha)+|\gamma|(1-\beta)]} \Gamma_{n}\right]^{1 /(n-1)}
$$

Proof. Let $f$ belong to $T$. It is known [20] that $f$ is close-to-convex of order $\delta$, if it satisfies the condition

$$
\left|f^{\prime}(z)-1\right|<1-\delta
$$

For the left-hand side of (3.2) we have

$$
\left|f^{\prime}(z)-1\right| \leq \sum_{n=2}^{\infty} n\left|a_{n}\right||z|^{n-1}
$$

The last expression is less than $1-\delta$ if

$$
\sum_{n=2}^{\infty} \frac{n}{1-\delta}\left|a_{n} \| z\right|^{n-1}<1
$$


International Journal of Mathematics and Mathematical Sciences

Using the fact that $f \in T S_{m}^{l}(\alpha, \beta, \gamma)$ if and only if

$$
\sum_{n=2}^{\infty} \frac{[(n+|\gamma|)(1-\beta)-(\alpha-\beta)]}{(1-\alpha)+|\gamma|(1-\beta)} \Gamma_{n}\left|a_{n}\right|<1
$$

we can say that (3.2) is true if

$$
\frac{n}{1-\delta}|z|^{n-1} \leq \frac{[(n+|\gamma|)(1-\beta)-(\alpha-\beta)]}{(1-\alpha)+|\gamma|(1-\beta)} \Gamma_{n}
$$

Or, equivalently,

$$
|z| \leq\left[\frac{(1-\delta)[(n+|\gamma|)(1-\beta)-(\alpha-\beta)]}{n[(1-\alpha)+|\gamma|(1-\beta)]} \Gamma_{n}\right]^{1 /(n-1)}
$$

which completes the proof.

Theorem 3.2. Let $f \in T S_{m}^{l}(\alpha, \beta, \gamma)$. Then the following are given.

(1) $f$ is starlike of order $\delta(0 \leq \delta<1)$ in the disc $|z|<r_{2}$, where

$$
r_{2}=\inf _{n \geq 2}\left\{\frac{(1-\delta)}{(n-\delta)} \frac{[(n+|\gamma|)(1-\beta)-(\alpha-\beta)]}{[(1-\alpha)+|\gamma|(1-\beta)]} \Gamma_{n}\right\}^{1 /(n-1)}
$$

(2) $f$ is convex of order $\delta(0 \leq \delta<1)$ in the unit disc $|z|<r_{3}$, where

$$
r_{3}=\inf _{n \geq 2}\left\{\frac{(1-\delta)}{n(n-\delta)} \frac{[(n+|\gamma|)(1-\beta)-(\alpha-\beta)]}{[(1-\alpha)+|\gamma|(1-\beta)]} \Gamma_{n}\right\}^{1 /(n-1)}
$$

Each of these results is sharp for the extremal function $f$ given by (2.10).

Proof. Let $f \in T$. It is known [1] that $f$ is starlike of order $\delta$, if it satisfies the condition

$$
\left|\frac{z f^{\prime}(z)}{f(z)}-1\right|<1-\delta
$$

For the left-hand side of (3.10) we have

$$
\left|\frac{z f^{\prime}(z)}{f(z)}-1\right| \leq \frac{\sum_{n=2}^{\infty}(n-1)\left|a_{n}\right||z|^{n-1}}{1-\sum_{n=2}^{\infty}\left|a_{n}\right||z|^{n-1}}
$$


The last expression is less than $1-\delta$ if

$$
\sum_{n=2}^{\infty} \frac{n-\delta}{1-\delta}\left|a_{n}\right||z|^{n-1}<1
$$

Using the fact that $f \in T S_{m}^{l}(\alpha, \beta, \gamma)$ if and only if

$$
\sum_{n=2}^{\infty} \frac{[(n+|\gamma|)(1-\beta)-(\alpha-\beta)]}{(1-\alpha)+|\gamma|(1-\beta)} \Gamma_{n}\left|a_{n}\right|<1
$$

we can say that $(3.10)$ is true if

$$
\frac{n-\delta}{1-\delta}|z|^{n-1}<\frac{[(n+|\gamma|)(1-\beta)-(\alpha-\beta)]}{(1-\alpha)+|\gamma|(1-\beta)} \Gamma_{n}
$$

Or, equivalently,

$$
|z|^{n-1}<\frac{(1-\delta)[(n+|\gamma|)(1-\beta)-(\alpha-\beta)]}{(n-\delta)[(1-\alpha)+|\gamma|(1-\beta)]} \Gamma_{n}
$$

which yields the starlikeness of the family.

Using the fact that $f$ is convex if and only if $z f^{\prime}$ is starlike, we can prove (2), on lines similar to those the proof of (1).

\section{Integral Means}

In order to find the integral means inequality and to verify the Silverman Conjuncture [21] for $f \in T S_{m}^{l}(\alpha, \beta, \gamma)$ we need the following subordination result due to Littlewood [22].

Lemma 4.1 (see [22]). If the functions $f$ and $g$ are analytic in $U$ with $g<f$, then

$$
\int_{0}^{2 \pi}\left|g\left(r e^{i \theta}\right)\right|^{\eta} d \theta \leq \int_{0}^{2 \pi}\left|f\left(r e^{i \theta}\right)\right|^{\eta} d \theta, \quad \eta>0, \quad z=r e^{i \theta}, 0<r<1 .
$$

Applying Theorem 2.1 with the extremal function and Lemma 4.1, we prove the following theorem.

Theorem 4.2. Let $\eta>0$. If $f \in T S_{m}^{l}(\alpha, \beta, \gamma)$ and $\{\Phi(\alpha, \beta, \gamma, n)\}_{n=2}^{\infty}$ is nondecreasing sequence, then, for $z=r e^{i \theta}$ and $0<r<1$, one has

$$
\int_{0}^{2 \pi}\left|f\left(r e^{i \theta}\right)\right|^{\eta} d \theta \leq \int_{0}^{2 \pi}\left|f_{2}\left(r e^{i \theta}\right)\right|^{\eta} d \theta
$$

where $f_{2}(z)=z-((1-\alpha)+|\gamma|(1-\beta)) z^{2} / \Phi(\alpha, \beta, \gamma, 2)$, and $\Phi(\alpha, \beta, \gamma, n)=[(n+|\gamma|)(1-\beta)-(\alpha-\beta)] \Gamma_{n}$. 
Proof. Let $f$ of the form of (1.2) and $f_{2}(z)=z-((1-\alpha)+|\gamma|(1-\beta)) z^{2} / \Phi(\alpha, \beta, \gamma, 2)$, then we must show that

$$
\int_{0}^{2 \pi}\left|1-\sum_{n=2}^{\infty} a_{n} z^{n-1}\right|^{\eta} d \theta \leq \int_{0}^{2 \pi}\left|1-\frac{(1-\alpha)+|\gamma|(1-\beta)}{\Phi(\alpha, \beta, \gamma, 2)} z\right|^{\eta} d \theta .
$$

By Lemma 4.1, it suffices to show that

$$
1-\sum_{n=2}^{\infty}\left|a_{n}\right| z^{n-1} \prec 1-\frac{(1-\alpha)+|\gamma|(1-\beta)}{\Phi(\alpha, \beta, \gamma, 2)} z
$$

Setting

$$
1-\sum_{n=2}^{\infty}\left|a_{n}\right| z^{n-1}=1-\frac{(1-\alpha)+|\gamma|(1-\beta)}{\Phi(\alpha, \beta, \gamma, 2)} w(z)
$$

from (4.5) and (2.1) we obtain

$$
\begin{aligned}
|w(z)| & =\left|\sum_{n=2}^{\infty} \frac{\Phi(\alpha, \beta, \gamma, n)}{(1-\alpha)+|\gamma|(1-\beta)} a_{n} z^{n-1}\right| \\
& \leq|z| \sum_{n=2}^{\infty} \frac{\Phi(\alpha, \beta, \gamma, n)}{(1-\alpha)+|\gamma|(1-\beta)}\left|a_{n}\right| \\
& \leq|z|<1 .
\end{aligned}
$$

This completes the proof of Theorem 4.2.

\section{Inclusion Relations Involving $N_{\delta}(e)$}

To study about the inclusion relations involving $N_{\delta}(e)$ we need the following definitions due to Goodman [23] and Ruscheweyh [24]. The $n, \delta$ neighborhood of function $f \in T$ is given by

$$
N_{\delta}(f)=\left\{g \in T: g(z)=z-\sum_{n=2}^{\infty}\left|b_{n}\right| z^{n}, \sum_{n=2}^{\infty} n\left|a_{n}-b_{n}\right| \leq \delta\right\} .
$$

Particularly for the identity function $e(z)=z$, we have

$$
N_{\mathcal{\delta}}(e)=\left\{g \in T: g(z)=z-\sum_{n=2}^{\infty}\left|b_{n}\right| z^{n}, \sum_{n=2}^{\infty} n\left|b_{n}\right| \leq \delta\right\} .
$$


Theorem 5.1. Let

$$
\delta=\frac{2[(1-\alpha)+|\gamma|(1-\beta)]}{[(2+|\gamma|)(1-\beta)-(\alpha-\beta)] \Gamma_{2}}
$$

where $\Gamma_{2}=\left(\alpha_{1} \cdots \alpha_{2}\right) /\left(\beta_{1} \cdots \beta_{2}\right)$. Then $\operatorname{TS}_{m}^{l}(\alpha, \beta, \gamma) \subset N_{\delta}(e)$.

Proof. For $f \in T S_{m}^{l}(\alpha, \beta, \gamma)$, Theorem 2.1 yields

$$
[(2+|\gamma|)(1-\beta)-(\alpha-\beta)] \Gamma_{2} \sum_{n=2}^{\infty}\left|a_{n}\right| \leq(1-\alpha)+|\gamma|(1-\beta)
$$

so that

$$
\sum_{n=2}^{\infty}\left|a_{n}\right| \leq \frac{(1-\alpha)+|\gamma|(1-\beta)}{[(2+|\gamma|)(1-\beta)-(\alpha-\beta)] \Gamma_{2}}
$$

On the other hand, from (2.1) and (5.5) we have

$$
\begin{aligned}
(1-\beta) \Gamma_{2} \sum_{n=2}^{\infty} n\left|a_{n}\right| \leq & (1-\alpha)+|\gamma|(1-\beta)+[(\alpha-\beta)-|\gamma|(1-\beta)] \Gamma_{2} \sum_{n=2}^{\infty}\left|a_{n}\right| \\
\leq & (1-\alpha)+|\gamma|(1-\beta)+[(\alpha-\beta)-|\gamma|(1-\beta)] \\
& \times \Gamma_{2} \frac{(1-\alpha)+|\gamma|(1-\beta)}{[(2+|\gamma|)(1-\beta)-(\alpha-\beta)] \Gamma_{2}} \\
\leq & \frac{[(1-\alpha)+|\gamma|(1-\beta)] 2(1-\beta)}{[(2+|\gamma|)(1-\beta)-(\alpha-\beta)]} \\
\sum_{n=2}^{\infty} n\left|a_{n}\right| \leq & \frac{2[(1-\alpha)+|\gamma|(1-\beta)]}{[(2+|\gamma|)(1-\beta)-(\alpha-\beta)] \Gamma_{2}} .
\end{aligned}
$$

Now we determine the neighborhood for each of the class $T S_{m}^{l}(\alpha, \beta, \gamma)$ which we define as follows. A function $f \in T$ is said to be in the class $T S_{m}^{l}(\alpha, \beta, \gamma)$ if there exists a function $g \in T S_{m}^{l}(\alpha, \beta, \gamma)$ such that

$$
\left|\frac{f(z)}{g(z)}-1\right|<1-\eta, \quad(z \in U, 0 \leq \eta<1) .
$$

Theorem 5.2. If $g \in T S_{m}^{l}(\alpha, \beta, \gamma)$ and

$$
\eta=1-\frac{\delta[(2+|\gamma|)(1-\beta)-(\alpha-\beta)] \Gamma_{2}}{2\left[((2+|\gamma|)(1-\beta)-(\alpha-\beta)) \Gamma_{2}-((1-\alpha)+|\gamma|(1-\beta))\right]},
$$

where $\Gamma_{2}=\left(\alpha_{1} \cdots \alpha_{2}\right) /\left(\beta_{1} \cdots \beta_{2}\right)$, then $N_{\delta}(g) \subset T S_{m}^{l}(\alpha, \beta, \gamma, \eta)$. 
Proof. Suppose that $f \in N_{\delta}(g)$, then we find from (5.6) that

$$
\sum_{n=2}^{\infty}\left|a_{n}-b_{n}\right| \leq \delta
$$

which implies the coefficient inequality

$$
\sum_{n=2}^{\infty}\left|a_{n}-b_{n}\right| \leq \frac{\delta}{2}
$$

Next, since $g \in T S_{m}^{l}(\alpha, \beta, \gamma)$, we have

$$
\sum_{n=2}^{\infty}\left|b_{n}\right| \leq \frac{2[(1-\alpha)+|\gamma|(1-\beta)]}{[(2+|\gamma|)(1-\beta)-(\alpha-\beta)] \Gamma_{2}}
$$

so that

$$
\begin{aligned}
\left|\frac{f(z)}{g(z)}-1\right| & <\frac{\sum_{n=2}^{\infty}\left|a_{n}-b_{n}\right|}{1-\sum_{n=2}^{\infty}\left|b_{n}\right|} \\
& \leq \frac{\delta}{2} \times \frac{[(2+|\gamma|)(1-\beta)-(\alpha-\beta)] \Gamma_{2}}{\left[((2+|\gamma|)(1-\beta)-(\alpha-\beta)) \Gamma_{2}-((1-\alpha)+|\gamma|(1-\beta))\right]} \\
& \leq 1-\eta,
\end{aligned}
$$

provided that $\eta$ is given precisely by (5.8). Thus by definition, $f \in T S_{m}^{l}(\alpha, \beta, \gamma, \eta)$ for $\eta$ given by (5.8), which completes the proof.

\section{Concluding Remarks}

By suitably specializing the various parameters involved in Theorems 2.1 to 5.2, we can state the corresponding results for the new subclasses defined in Examples 1.1 to 1.4 and also for many relatively more familiar function classes.

\section{Acknowledgment}

The authors would like to record their sincerest thanks to the referee(s) for their valuable suggestions and comments.

\section{References}

[1] H. Silverman, "Univalent functions with negative coefficients," Proceedings of the American Mathematical Society, vol. 51, pp. 109-116, 1975.

[2] S. Ruscheweyh, "New criteria for univalent functions," Proceedings of the American Mathematical Society, vol. 49, pp. 109-115, 1975. 
[3] J. Dziok and H. M. Srivastava, "Certain subclasses of analytic functions associated with the generalized hypergeometric function," Integral Transforms and Special Functions, vol. 14, no. 1, pp. 7-18, 2003.

[4] B. C. Carlson and D. B. Shaffer, "Starlike and prestarlike hypergeometric functions," SIAM Journal on Mathematical Analysis, vol. 15, no. 4, pp. 737-745, 1984.

[5] S. Owa, "On the distortion theorems. I," Kyungpook Mathematical Journal, vol. 18, no. 1, pp. 53-59, 1978.

[6] H. M. Srivastava and S. Owa, "Some characterization and distortion theorems involving fractional calculus, generalized hypergeometric functions, Hadamard products, linear operators, and certain subclasses of analytic functions," Nagoya Mathematical Journal, vol. 106, pp. 1-28, 1987.

[7] A. W. Goodman, "On uniformly convex functions," Annales Polonici Mathematici, vol. 56, no. 1, pp. 87-92, 1991.

[8] A. W. Goodman, "On uniformly starlike functions," Journal of Mathematical Analysis and Applications, vol. 155, no. 2, pp. 364-370, 1991.

[9] F. Rønning, “Uniformly convex functions and a corresponding class of starlike functions," Proceedings of the American Mathematical Society, vol. 118, no. 1, pp. 189-196, 1993.

[10] F. Rønning, "Integral representations of bounded starlike functions," Annales Polonici Mathematici, vol. 60, no. 3, pp. 289-297, 1995.

[11] S. Kanas and A. Wisniowska, "Conic regions and $k$-uniform convexity," Journal of Computational and Applied Mathematics, vol. 105, no. 1-2, pp. 327-336, 1999.

[12] S. Kanas and A. Wiśniowska, "Conic domains and starlike functions," Revue Roumaine de Mathematiques Pures et Appliquees, vol. 45, no. 4, pp. 647-657, 2000.

[13] S. Kanas and H. M. Srivastava, "Linear operators associated with $k$-uniformly convex functions," Integral Transforms and Special Functions, vol. 9, no. 2, pp. 121-132, 2000.

[14] S. Kanas and T. Yaguchi, "Subclasses of $k$-uniformly convex and starlike functions defined by generalized derivative. II," Publications Institut Mathematique, vol. 69(83), pp. 91-100, 2001.

[15] O. Altintas, O. Ozkan, and H. M. Srivastava, "Neighborhoods of a class of analytic functions with negative coefficients," Applied Mathematics Letters, vol. 13, no. 3, pp. 63-67, 2000.

[16] G. Murugusundaramoorthy and H. M. Srivastava, "Neighborhoods of certain classes of analytic functions of complex order," Journal of Inequalities in Pure and Applied Mathematics, vol. 5, no. 2, article 24, pp. 1-8, 2004

[17] G. Murugusundaramoorthy and N. Magesh, "Starlike and convex functions of complex order involving the Dziok-Srivastava operator," Integral Transforms and Special Functions, vol. 18, no. 5-6, pp. 419-425, 2007.

[18] S. D. Bernardi, "Convex and starlike univalent functions," Transactions of the American Mathematical Society, vol. 135, pp. 429-446, 1969.

[19] G. Murugusundaramoorthy and N. Magesh, "Linear operators associated with a subclass of uniformly $\beta$-convex functions," in Proceedings of the National Conference on Geometry, Analysis, Fluid Mechanics and Computer Applications, pp. 103-110, Kuvempu University, Karnataka, India, December 2004.

[20] H. S. Al-Amiri, "On a subclass of close-to-convex functions with negative coefficients," Mathematica, vol. 31(54), no. 1, pp. 1-7, 1989.

[21] H. Silverman, "Integral means for univalent functions with negative coefficients," Houston Journal of Mathematics, vol. 23, no. 1, pp. 169-174, 1997.

[22] J. E. Littlewood, "On inequalities in theory of functions," Proceedings of the London Mathematical Society, vol. 23, pp. 481-519, 1925.

[23] A. W. Goodman, "Univalent functions and nonanalytic curves," Proceedings of the American Mathematical Society, vol. 8, pp. 598-601, 1957.

[24] S. Ruscheweyh, "Neighborhoods of univalent functions," Proceedings of the American Mathematical Society, vol. 81, no. 4, pp. 521-527, 1981. 


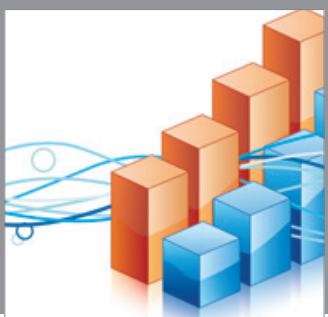

Advances in

Operations Research

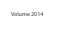

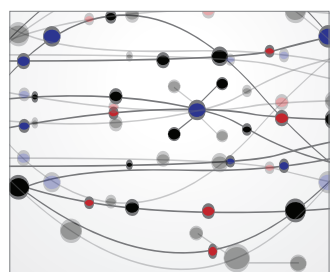

\section{The Scientific} World Journal
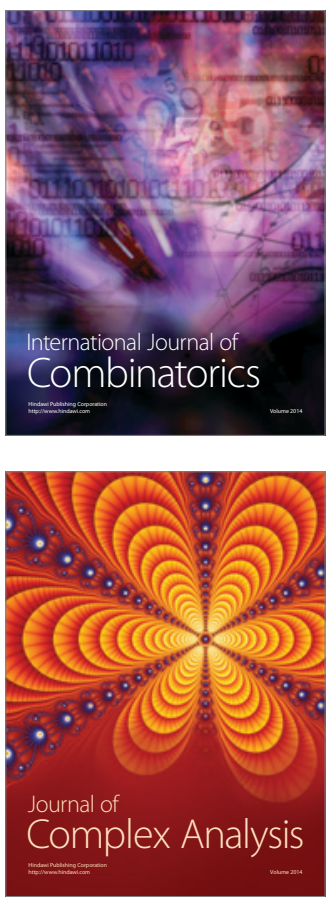

International Journal of

Mathematics and

Mathematical

Sciences
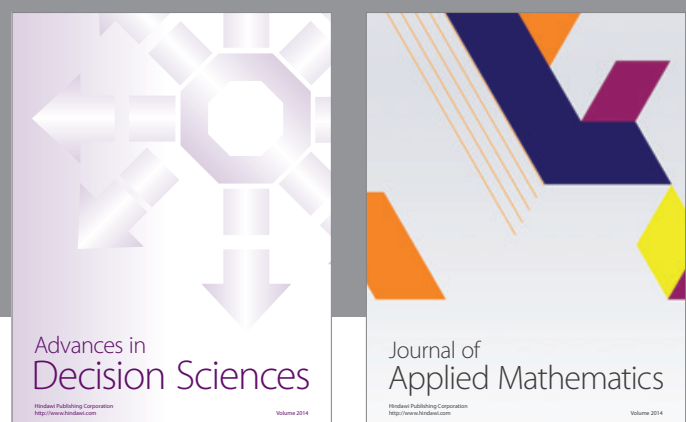

Journal of

Applied Mathematics
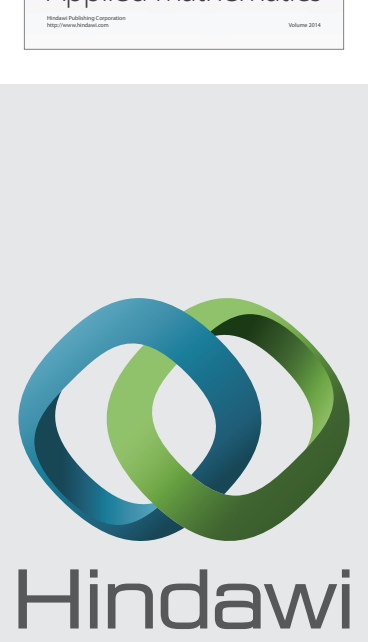

Submit your manuscripts at http://www.hindawi.com
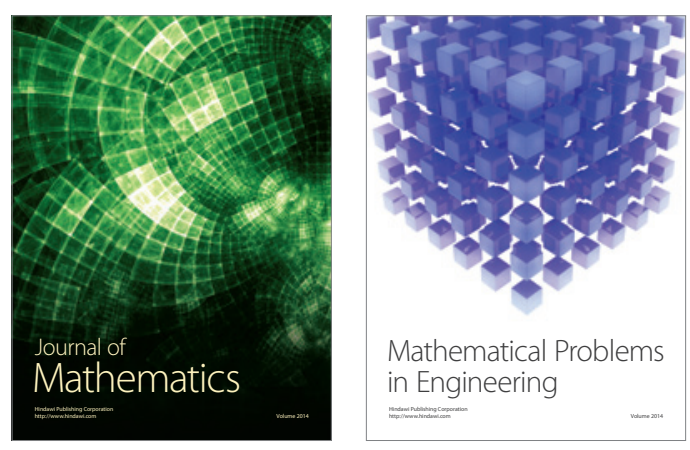

Mathematical Problems in Engineering
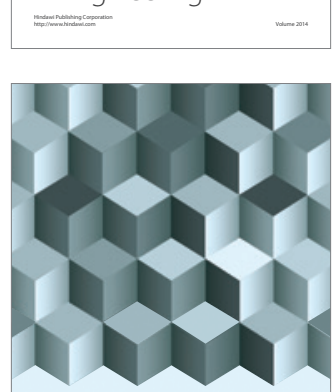

Journal of

Function Spaces
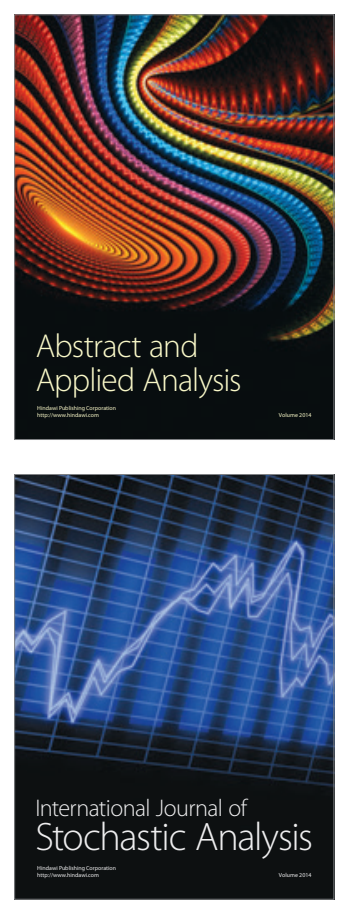

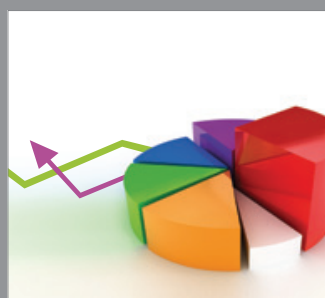

ournal of

Probability and Statistics

Promensencen
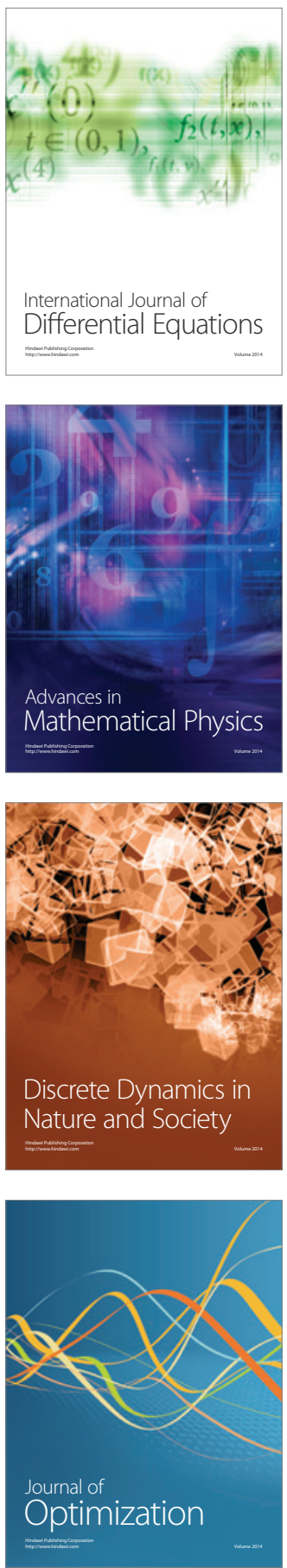